\title{
Parâmetros genéticos e fenotípicos do desempenho reprodutivo de fêmeas Chianina
}

\section{Danielle Maria Machado Ribeiro Azevêdo', Raimundo Martins Filho², Riccardo Bozzi ${ }^{3}$, Flavio Forabos $\mathrm{Co}^{4}$, Carlos Henrique Mendes Malhado ${ }^{5}$}

\author{
${ }^{1}$ Embrapa Meio-Norte. \\ 2 Doutor, Bolsista CNPq/FAPEPI/UFPI. \\ 3 Professor visitante - DZ/UFC. \\ ${ }^{4}$ Associazione Nazionale Allevatori Bovini Italiani da Carne (ANABIC). \\ 5 UESB.
}

RESUMO - Objetivou-se estimar os parâmetros genéticos e fenotípicos de características reprodutivas de fêmeas bovinas da raça Chianina criadas em diferentes rebanhos participantes da Associazione Nazionale Allevatori Bovini Italiani da Carne (ANABIC). As características estudadas foram idade ao primeiro parto (IPP), primeiro intervalo de partos (IDP1) e intervalo médio de partos (IMDP). As análises estatísticas foram realizadas pelo procedimento General Linear Model (GLM) do programa estatístico SAS (Statistical Analysis System) e os componentes de variância foram estimados pelo método de máxima verossimilhança restrita utilizando-se o software MTDFREML sob modelo animal. Os números de observações utilizados para IPP, IDP1 e IMDP foram, respectivamente, 31.023; 23.998 e 94.497 e as médias encontradas, em dias, $1.037,69 \pm 186,37 ; 457,93 \pm 96,80$ e 436,26 \pm 90,83 para IPP, IDP1, IDPM. Todas as características avaliadas foram influenciadas pelo rebanho. Verificou-se efeito de estação e ano de nascimento da vaca sobre a IPP. O IDP1 e o IDPM foram influenciados por rebanho, estação e ano do parto precedente, observando-se efeito também da ordem de parição sobre o IDPM. As estimativas de herdabilidade para IPP, IDP1 e IDPM foram, respectivamente de 0,36 $\pm 0,014 ; 0,13 \pm 0,014$ e $0,05 \pm 0,004$. A repetibilidade para IDPM foi de $0,075 \pm 0,004$. A utilização de IPP e IDP1 em programas de melhoramento genético pode resultar em maior precocidade e mais alto potencial para longevidade nestes rebanhos.

Palavras-chave: herdabilidade, idade ao primeiro parto, intervalo de partos, repetibilidade

\section{Genetic and phenotypic parameters of reproductive performance of Chianina females}

\begin{abstract}
This study aimed to evaluate the reproductive performance of Chianina cows born from 1977 to 2002. Data was used to calculate age at first calving (AFC), first calving interval (CI1) and average calving intervals (avgCIs) for the whole lifetime of cows. After editing data, the number of records used for AFC, CI1 and avgCIs analyses were respectively 31,023; 23,998 and 94,497 respectively. Statistical analyses were done using the SAS program (Statistical Analysis System) and variance components were estimated by REML using the software MTDFREML fitting animal models. Means for AFC, CI1 and avgCIs were 1,037.69 $\pm 186.37,457.93 \pm 12.22$ and 436,26 $\pm 12,17$ days, respectively. Heritability estimates for $\mathrm{AFC}, \mathrm{CI} 1$ and avgCIs were respectively $0.36 \pm 0.014 ; 0.13 \pm 0.014$ e $0.05 \pm 0.004$ and the repeatability for avgCIs was 0.075 \pm 0.004 . The use of AFC and CI1 in genetic improvement programs may lead to an increase in precocity and potential longevity. Changes in management of females may decrease avgCIs, leading to higher and faster improvement in reproductive efficiency.
\end{abstract}

Key Words: age at first calving, calving interval, heritability, repeatability

\section{Introdução}

A raça Chianina é considerada autóctone da Itália, sendo uma das mais importantes raças para produção de carne. Essa raça vem sendo difundida em nível internacional desde a II Guerra Mundial (ARSIA, 2002). No Brasil, a raça Chianina desperta o interesse de produtores e pesquisadores, principalmente por suas características de produção, seja como raça pura, seja como raça terminal em cruzamentos com raças indianas ou européias. Neste contexto, os resultados encontrados têm sido alentadores (Villares, 1975; Zamorana Bucio, 1979; Pereira \& Silva, 1982; Madalena et al., 1989; Pádua \& Silva, 1994; Martini et al., 1996; Euclides Filho et al., 1997a,b, 1998).

As características reprodutivas da raça Chianina, relacionadas à precocidade, como a idade ao primeiro parto, ou 
aquelas relacionadas à periodicidade reprodutiva, como o intervalo de partos, têm sido pouco estudadas no Brasil ou mesmo em seu país de origem.

Alguns estudos foram conduzidos no Brasil com fêmeas Chianina puras (Villares, 1975; Biffani, 1999; Biffani et al., 2000) e mestiças (Villares, 1975; Silva et al., 1979a,b; Silva \& Pereira, 1986a,b), mas, como é reduzido o plantel existente no país, é pequeno o número de informações. Biffani et al. (2000) afirmaram que, apesar do número limitado de dados utilizado em seu trabalho, a raça Chianina pode ser uma opção viável para a produção de carne em ambientes tropicais.

$\mathrm{Na}$ Itália foram realizados alguns trabalhos utilizando informações da Associazione Allevatori Bovini Italiani da Carne (ANABIC) (Bozzi et al., 1998; Franci et al., 1998; Filippini \& Forabosco, 2001), nos quais foi demonstrado que a raça Chianina é menos precoce em termos reprodutivos que outras raças taurinas de clima temperado, provavelmente em virtude de sua maior capacidade de adaptação a ambientes adversos como os de algumas províncias italianas.

A avaliação das características reprodutivas de uma raça em seu ambiente natural, ou seja, na região onde foi formada, é uma das melhores formas de se conhecer o potencial da raça, visto que, nesse ambiente, espera-se que esteja totalmente adaptada. Esse conhecimento pode ser utilizado como parâmetro para avaliação da adaptação da raça em outras regiões de condições ambientais semelhantes às do país de origem da raça, mesmo que apenas em determinadas épocas do ano.

Os objetivos neste trabalho foram analisar, a partir de informações da raça Chianina em seu país de origem, a Itália, as características reprodutivas consideradas de interesse pela ANABIC e verificar a possibilidade de contribuição para os processos de seleção desta raça.

\section{Material e Métodos}

Foram analisados dados de fêmeas da raça Chianina pertencentes a rebanhos localizados em diversas províncias italianas e participantes da Associazione Nazionale Allevatori Bovini Italiani da Carne (ANABIC). Os animais eram criados, predominantemente, de forma intensiva em pequenos rebanhos, recebendo concentrado e forragem no cocho. Nos poucos rebanhos de grande porte, a criação era feita a pasto, de forma extensiva.

Analisaram-se apenas a idade ao primeiro parto (IPP), o primeiro intervalo de partos (IDP1) e o intervalo médio de partos (IMDP), por não constar no controle reprodutivo realizado pela ANABIC a data da inseminação artificial ou monta controlada, não sendo possível estimar o período de serviço, o número de serviços por concepção e o período de gestação.

Os dados coletados foram enviados à ANABIC, duas ou três vezes por ano, via internet $(99 \%)$. O banco de dados foi editado e testado eliminando-se todos os dados fora dos limites impostos, de 900 a 2.561 dias para idade ao primeiro parto e de 321 a 863 dias para os intervalos de parto. Ao final destas restrições, permaneceram para IPP, IDP1 e IDPM, respectivamente, $31.023,23.998$ e 94.497 informações.

O modelo animal proposto para análise dos componentes de variância para as características IPP e IDP1 continha o efeito aleatório do animal e os efeitos fixos. O modelo geral para a característica IDPM consistiu de efeitos aleatórios (animal e ambiente permanente) e de efeitos fixos. Os efeitos fixos variaram de acordo com a característica: a) IPP rebanho, ano e estação de nascimento da vaca e sexo da cria; b) IDP1 - rebanho, estação e ano do parto precedente e sexo da cria; e c) IDPM - variáveis do IDP1 e, ainda, ordem de parição. Foram consideradas quatro estações de nascimento ou acasalamento, definindo-se os trimestres em: 1 - janeiro a março, 2 - abril a junho, 3 - julho a setembro e 4 - outubro a dezembro.

Os componentes de variância necessários à obtenção dos parâmetros genéticos foram estimados por meio do modelo animal, pelo método de máxima verossimilhança restrita não derivativa (DFREML - Derivative-Free Restricted Maximum Likelihood), pela aplicação do software MTDFREML (Multiple Trait Derivative-Free Restricted Maximum Likelihood), de acordo com Boldman et al. (1995).

\section{Resultados e Discussão}

A idade ao primeiro parto (IPP) é fundamental na eficiência da pecuária bovina e sua redução contribui para diminuir os custos de produção, visto que as fêmeas de reposição iniciam mais cedo a vida reprodutiva. A IPP, pela praticidade de mensuração e por estar relacionada à longevidade potencial da fêmea, é uma das medidas utilizadas em programas de melhoramento genético com o objetivo de avaliar a eficiência reprodutiva dos rebanhos.

O valor encontrado para IPP neste trabalho foi de $1.037,69 \pm 186,37$ dias (Tabela 1) e está próximo aos resultados citados por Bozzi et al. (1998) e Franci et al. (1998), que encontraram, respectivamente, $1.017,97$ e 1.020,1 dias para fêmeas Chianina criadas na Itália. Entretanto, é superior aos 984,4 dias obtidos em 21.365 observações por Filippini \& Forabosco (2001), também em rebanhos italianos da raça Chianina. Esses autores utilizaram dados de fêmeas nascidas após 1984, enquanto, neste estudo, foram consideradas 
Tabela 1 - Número de observações $(\mathrm{N})$, média observada, desvio-padrão (DP) e coeficiente de variação (CV, \%) para as características estudadas

Table 1 - Number of records (N), means, standard deviation (s.d.) and coefficient at variation (CV) for reproductive traits

\begin{tabular}{lccc}
\hline & IPP (dias) & IDP 1 (dias) & IDP M (dias) \\
& $A F C$ (days) & CII (days) & avgCIs (days) \\
\hline $\mathrm{N}$ & 31.023 & 23.998 & 94.497 \\
Média (Mean) & $1.037,69$ & 457,93 & 436,26 \\
$\mathrm{DP}$ (SD) & 186,37 & 96,81 & 90,83 \\
$\mathrm{CV}$ & 17,97 & 21,14 & 20,82 \\
\hline
\end{tabular}

IPP = idade ao primeiro parto; IDP 1 = primeiro intervalo de partos; IDP $\mathrm{M}=$ intervalo médio de partos.

$A F C=$ age at first calving; $C / 1$ = first calving interval; avgC/s = average calving intervals.

informações de fêmeas nascidas a partir de 1977, ou seja, oito anos antes. Provavelmente, os diferentes resultados podem ser decorrentes da seleção para precocidade efetivada pela ANABIC.

No Brasil, alguns pesquisadores avaliaram a IPP de fêmeas Chianina puras ou mestiças (Villares, 1975; Alves Neto \& Pereira, 1978; Silva \& Pereira, 1986a,b; Biffani et al., 2000) no Centro-Oeste e Nordeste do país. Os autores encontraram resultados superiores ao obtido neste trabalho, exceto Biffani et al. (2000), que observaram a primeira parição aos 1.021,5 dias em 54 fêmeas Chianina puras criadas em Pernambuco, o que pode indicar adaptação da raça às condições tropicais locais.

A IPP das fêmeas Chianina foi influenciada $(\mathrm{P}<0,01)$ pelo ano e mês de nascimento da vaca e pelo rebanho, sem efeito do sexo da cria $(\mathrm{P}>0,05)$.

Considerando que fatores de ambiente, como clima, manejo nutricional, sanitário e reprodutivo podem influenciar a IPP, esperava-se que esta característica sofresse efeito do mês e ano de nascimento da vaca e da propriedade em que as fêmeas foram criadas. No entanto, esperava-se também que o sexo da cria interferisse na IPP, visto que a duração da gestação tende a ser reduzida quando a fêmea gesta uma cria do sexo feminino (Leite et al., 1986; Silva \& Pereira, 1986b; Zillo et al.,1986; Bacalhau et al., 1998; Azevêdo, 2003).
Em trabalhos realizados por outros autores, a IPP foi influenciada por ano e época do parto em fêmeas mestiças Chianina x Zebu (Silva \& Pereira, 1986a) e por ano de nascimento da vaca e interação ano x estação de nascimento da vaca em fêmeas Chianina puras (Biffani et al., 2000), semelhante ao encontrado neste trabalho.

Na Tabela 2 são descritos os parâmetros genéticos para as características reprodutivas estudadas. O valor estimado para herdabilidade da IPP $(0,36 \pm 0,014)$ foi o dobro do obtido por Franci et al. (1998), de 0,18 $\pm 0,02$, para fêmeas Chianina puras criadas na Itália. Entretanto, foi inferior aos $0,63 \pm 0,16$ encontrados por Silva \& Pereira (1986a) para fêmeas $1 / 2$ Chianina $x$ Zebu criadas no Brasil. A divergência entre estes resultados deve-se, provavelmente, às diferenças de ambiente entre rebanhos.

Apesar de as características reprodutivas possuírem, de modo geral, valores reduzidos de herdabilidade (Martins Filho et al., 1994; Lobo et al., 2000) e, por isso, serem pouco utilizadas como meio de seleção, a herdabilidade estimada para IPP neste trabalho, que pode ser considerada de magnitude moderada, corrobora a utilização desta característica em programas de melhoramento genético da raça Chianina, mesmo que melhorias no manejo dos animais possam ser alternativas mais rápidas para a redução em sua expressão. Este maior valor para herdabilidade em relação a outras características reprodutivas, como o intervalo de partos, por exemplo, advém da sua inter-relação com a idade à puberdade, que possui grande influência da herança genética aditiva e, portanto, herdabilidade relativamente alta (MacNeil et al., 1984).

Uma conseqüência favorável da seleção para precocidade em relação à IPP seria a maior longevidade potencial da fêmea no rebanho e, conseqüentemente, os menores custos com fêmeas na fase de recria, período no qual não estão produzindo bezerros, mas necessitam de alimentação, o que pode onerar os custos de produção na propriedade.

O intervalo de partos (IDP) é um dos aspectos mais relevantes para a reprodução, pois trata-se de um fator

Tabela 2 - Componentes de variância e parâmetros genéticos das características reprodutivas estudadas Table 2 - Estimates of variance components, heritability and repeatability for reproductive traits

\begin{tabular}{lccccccc}
\hline $\begin{array}{l}\text { Característica } \\
\text { Trait }\end{array}$ & $\sigma_{\mathrm{a}}^{2}$ & $\sigma_{\mathrm{e}}^{2}$ & $\sigma_{\mathrm{ep}}^{2}$ & $\sigma_{\mathrm{f}}^{2}$ & $\mathrm{~h}^{2}$ & $\mathrm{r}$ \\
\hline IPP (AFC) & $12.943,45$ & $23.070,49$ & - & $36.013,94$ & $0,36 \pm 0,014$ & - \\
IDP1 (CII) & $1.240,52$ & $8.240,29$ & - & $9.480,81$ & $0,13 \pm 0,014$ & - \\
IDPM (ageCIs) & 412,52 & $7.692,24$ & 213,39 & $8.318,16$ & $0,05 \pm 0,004$ & $0,075 \pm 0,004$ \\
\hline
\end{tabular}

IPP = idade ao primeiro parto; IDP1 = primeiro intervalo de partos; IDPM = intervalo de partos médio; $\sigma^{2}$ = variância genética aditiva; $\sigma^{2}{ }_{\mathrm{e}}=$ variância de ambiente $\sigma^{2}$ e $=$ variância de ambiente permanente; $\sigma_{\mathrm{f}}^{2}=$ variância fenotípica; $\mathrm{h}^{2}=$ herdabilidade; $\mathrm{r}=$ repetibilidade

$A F C=$ age at first calving; $C / 1=$ first calving interval; avg Cls = average calving intervals; $\sigma_{a}^{2}=$ genetic additive variance; $\sigma_{e}^{2}=$ residual variance; $\sigma_{e p}^{2}=$ permanent environment variance; $\sigma_{f}^{2}=$ phenotipic variance; $h^{2}=$ heritability; $r=$ repeatibility . 
preponderante na eficiência reprodutiva e no rendimento econômico da pecuária bovina.

Os rebanhos incluídos nesta análise apresentaram IDP1 e IDPM de 457,93 \pm 96,80 e 436,26 \pm 90,83 dias, respectivamente (Tabela 1 ).

O IDP1 foi superior ao IDPM, fato já documentado na literatura para mestiços $1 / 2$ Chianina x Zebu (Silva \& Pereira, 1986b), para fêmeas Chianina puras (Bozzi et al., 1998; Franci et al., 1998) e para fêmeas da raça Nelore (Mercadante et al., 2000; Pereira et al., 2002; Simioni, 2002). A maior duração do IDP1 decorreu, provavelmente, do fato de a fêmea em primeira ordem de parto ser mais exigente em termos nutricionais por não ter atingido ainda seu desenvolvimento corporal completo, precisando consumir alimento para mantença, crescimento, amamentação e restabelecimento de seu eixo hipotalâmico-hipofisáriogonadal para que possa apresentar novo estro e ser fertilizada.

Villares (1975) analisou dados de matrizes Chianina, Guzerá e mestiças F1 destas raças e encontrou, respectivamente, 433,88; 483,03 e 508,83 dias para IDPM. Como se observa, a raça taurina apresentou o menor IDPM e as mestiças o maior. Esse autor ressaltou, no entanto, que a maioria das fêmeas Chianina x Guzerá estava ainda no segundo parto, ou seja, no IDP1, o que ocasionou IDPM superior ao dos outros grupos estudados.

Bozzi et al. (1998), utilizando dados da ANABIC, avaliaram o IDP de 20.876 informações de animais registrados na Associação e encontraram 450,85 \pm 97,38 dias para o IDP1. Franci et al. (1998), também trabalhando com dados da ANABIC, verificaram, respectivamente, $450,9 \pm 97,4 ; 433,9$ $\pm 89,5 ; 426,1 \pm 86,5$ e $421,8 \pm 84,8$ dias para o $1^{\circ} \stackrel{\circ}{ }, 2^{\circ}, 3^{\circ}$ e e $4^{\circ}$ intervalos de parto. Percebe-se, nitidamente, redução no IDP com o aumento da ordem de parição. O IDPM, obtido a partir de 80.599 informações, foi de $432,6 \pm 90,0$ dias, bem próximo ao encontrado neste trabalho.

Filippini \& Forabosco (2001), analisando dados da ANABIC, encontraram para o $1^{\underline{o}}, 2^{\underline{o}}$ e $3^{\circ}$ IDP, respectivamente, $448,8 \pm 96,2 ; 438,6 \pm 89,5$ e 432,2 $\pm 87,8$ dias, ou seja, também houve tendência de redução do IDP à medida que a ordem do parto aumentou.

Em estudo com animais da raça Chianina no Brasil, Biffani et al. (2000) observaram IDPM de 468,8 $\pm 170,36$ dias, superior ao das fêmeas Chianina criadas na Itália avaliadas neste trabalho, o que pode sugerir manejo inadequado no rebanho brasileiro.

O IDP1 e o IDPM sofreram influência significativa $(\mathrm{P}<0,01)$ do rebanho e da estação e ano do parto anterior. A ordem de parição também influenciou $(\mathrm{P}<0,01)$ a estação e o ano de parto anterior.
Sabe-se que variações climáticas, como a distribuição das chuvas e a amplitude térmica, ocorrem anualmente e podem influenciar a disponibilidade e qualidade das pastagens, ocasionando deficiência nutricional dos animais. No entanto, considerando que a maioria das propriedades era constituída de pequenos rebanhos e que os animais eram tratados em sistema semi-intensivo, com fornecimento de alimento no cocho durante todo o ano, não se esperava influência de fatores ambientais sobre as características IDP1 e IDPM. Provavelmente, mesmo com os animais em confinamento, pode ter havido certa variação estacional no fornecimento de alimentos capaz de influenciar as características estudadas; outras práticas de manejo, sanitárias ou reprodutivas, variáveis entre rebanhos e estação e ano do parto, podem ainda ter afetado o intervalo de partos. Quanto à ordem de parição, a qual influenciou o IDPM, os resultados confirmam os de outros autores (Villares, 1975; Franci et al., 1998) e podem ser conseqüência da incorporação de fêmeas primíparas ao rebanho, como discutido anteriormente.

Considerando que o período de gestação é pouco afetado por fatores ambientais, sendo praticamente constante, a influência da estação e do ano do parto pode estar incindindo sobre o período de serviço, causando efeito sobre o IDP. Portanto, quando o objetivo for a obtenção de 1 parto/vaca/ano, devem ser adotadas mudanças de manejo que possibilitem redução no período de serviço. Estas mudanças devem ter como base a suplementação alimentar nos períodos mais críticos do ano e o remanejamento das estações de acasalamento e nascimento para épocas em que a disponibilidade de alimento não for restritiva ao desempenho dos animais.

As estimativas de herdabilidade para IDP1 e IDPM são apresentadas na Tabela 2. Apesar do valor relativamente baixo para herdabilidade do IDP1 $(0,13 \pm 0,014)$, esta característica apresentou variabilidade suficiente para ser utilizada em programas de seleção, provavelmente em virtude da influência da IPP. No entanto, o melhor manejo, sobretudo alimentar, das novilhas pode reduzir o IDP1 de forma mais rápida que a seleção.

O reduzido valor estimado para a herdabilidade do $\operatorname{IDPM}(0,05 \pm 0,004)$ comprova que o ambiente é responsável por grande parte da expressão da característica e, portanto, alterações no manejo na propriedade, principalmente no aspecto nutricional, são medidas bem mais rápidas para se reduzir o IDPM e obter melhor ajuste na eficiência reprodutiva dos rebanhos.

A repetibilidade encontrada para o IDPM foi de 0,075 $\pm 0,004$ (Tabela 2) e indica que a permanência ou não de fêmeas no rebanho de reprodução não deve ser pautada 
apenas no IDP, pois o valor obtido tem pequena probabilidade de se repetir ao longo da vida produtiva da matriz.

\section{Conclusões}

As características idade ao primeiro parto e primeiro intervalo de partos para a raça Chianina possuem herdabilidades que permitem sua utilização em programas de melhoramento genético visando maior precocidade e longevidade do rebanho.

O intervalo médio de partos na raça Chianina sofre pouca influência da genética aditiva e, portanto, modificações no manejo são mais eficientes para a redução dessa característica.

\section{Literatura Citada}

ALVES NETO, F.; PEREIRA, G.A. Age at first parturition and inter-parturition interval of Chianina cows in Brazil. In: INTERNATIONAL CONGRESS OF THE CHIANINA BREED, 2., São Paulo. Proceedings... São Paulo: Associação Brasileira dos Criadores de Chianina, 1978. p.219-241.

AGENZIA REGIONALE PER LO SVILUPPO E L'INNOVAZIONE NEL SETTORE AGRICOLO-FORESTALE - ARSIA. Risorse genetiche animali autóctone della Toscana. Toscana: ARSIA, 2002. 165p.

AZEVÊDO, D.M.M.R. Avaliação genética e fenotípica de características reprodutivas e da produtividade acumulada de fêmeas Nelore nas regiões Norte e Nordeste do Brasil. Fortaleza: Universidade Federal do Ceará. 2003. 122p. Tese (Doutorado em Zootecnia) - Universidade Federal do Ceará, 2003.

BACAlHaU, A.S.; GUEDES, P.L.C.; RANGEL, A.H.N. et al. Fatores que influem no período de gestação de vacas Gir leiteiras. In: REUNIÃO ANUAL DA SOCIEDADE BRASILEIRA DE ZOOTECNIA, 35., 1998, Botucatu. Anais... Botucatu: Sociedade Brasileira de Zootecnia, 1998. CD-ROM.

BIFFANI, S. Caratteristiche riproduttive di femmine di razza Chianina e Nelore allevate nel Nord Este del Brasile. Firenze: Università degli Studi di Firenze, 1999. 104p. Tese (Doutorado em Scienze Zootecniche) - Università degli Studi di Firenze, 1999.

BIFFANI, S.; MARTINS FILHO, R.; LUCIFERO, M. et al. Parâmetros reprodutivos de fêmeas da raça Chianina criadas no estado do Pernambuco. In: REUNIÃO ANUAL DA SOCIEDADE BRASILEIRA DE ZOOTECNIA, 37., 2000, Viçosa, MG. Anais...Viçosa, MG: Sociedade Brasileira de Zootecnia, 2000. CD-ROM.

BOLDMAN, K.G.; KRIESE, L.A.; VAN VLECK, L.D. et al. A manual for use of MTDFREML - a set of programs to obtain estimates of variances and covariances (DRAFT). Lincoln: USDA-ARS, 1995. 120p.

BOZZI, R.; FRANCI, O.; BIFFANI, S. et al. Effect of data structure on estimates of genetic parameters in Chianina breed. Zootecnia Nutrition Animal, v.24, p.53-61, 1998.

EUCLIDES FILHO, K.; EUCLIDES, V.P.B.; FIGUEIREDO, G.R. et al. Avaliação de animais Nelore e de seus mestiços com Charolês, Fleckvieh e Chianina, em três dietas. 1. Ganho de peso e conversão alimentar. Revista Brasileira de Zootecnia, v.26, n.1, p.66-72, 1997a.

EUCLIDES FILHO, K.; EUCLIDES, V.P.B.; FIGUEIREDO, G.R. et al. Avaliação de animais Nelore e de seus mestiços com
Charolês, Fleckvieh e Chianina, em três dietas. 2. Características de carcaça. Revista Brasileira de Zootecnia, v.26, n.1, p.73-79, 1997b

EUCLIDES FILHO, K.; FIGUEIREDO, G.R.; SILVA, L.O.C. et al Idade aos $165 \mathrm{~kg}$ de peso vivo para progênies Nelore, Fleckvieh, Chianina, Charolês, F1's e retrocruzas. Revista Brasileira de Zootecnia, v.27, n.5, p.899-905, 1998.

FILIPPINI, F.; FORABOSCO, F. The genetic evaluation of cows Taurus International, v.10, n.6, p.20-25, 2001

FRANCI, O.; PANELLA, F.; ACCIAIOLI, A. et al. Estimates of genetic parameters for some reproductive traits in Chianina beef cattle. Zootecnia Nutrition Animal, v.24, p.31-39, 1998

LEITE, P.R.M.; BELLIDO, M.M.; PACA, F.R. et al. Fatores que influenciam o período de gestação e intervalo entre partos de vacas Gir no Nordeste brasileiro. Pesquisa Agropecuária Brasileira, v.21, n.1, p.87-92, 1986

LÔBO, R.N.B.; MADALENA, F.E.; VIEIRA, A.R. Average estimates of parameters for beef and dairy cattle in tropical regions. Animal Breeding Abstracts, v.68, p.433-462, 2000.

MacNEIL, M.D.; CUNDIFF, L.V.; DINKEL, C.A. et al. Genetics correlations among sex-limited traits in beef cattle. Journal of Animal Science, v.58, p.1.171, 1984.

MADALENA, F.E.; SANTOS, A.J.R.; FREITAS, A.J Desenvolvimento de mestiço leiteiro brasileiro (MLB). 2. Comparação do crescimento ponderal de tourinos MLB e $1 / 4$ Chianina $x 3 / 4$ Nelore. Arquivo Brasileiro de Medicina Veterinária e Zootecnia, v.41, p.355-367, 1989.

MARTINI, A.A.; GIORGETTI, R; FUNGHI, R. et al. Cruzamento de vacas de raça Nelore com sêmen de touros da raça Chianina de dois diferentes tipos genéticos. Resultados em vida. In: REUNIÃO ANUAL DA SOCIEDADE BRASILEIRA DE ZOOTECNIA, 33., 1996, Fortaleza. Anais... Fortaleza: Sociedade Brasileira de Zootecnia, 1996. v.1, p.115-117.

MARTINS FILHO, R.; LÔBO, R.B. Correlações genéticas entre circunferência escrotal e características reprodutivas de fêmeas em bovinos da raça Nelore. Ciência Agronômica, v. 25, p. $10-15,1994$.

MERCADANTE, M.E.Z.; LÔBO, R.B.; OLIVEIRA, H.N Estimativas de (co)variâncias entre características de reprodução e de crescimento em fêmeas de um rebanho Nelore. Revista Brasileira de Zootecnia, v.29, n.4, p.997-1004, 2000.

PÁDUA, J.T.; SILVA, R.G. Avaliação genética do desempenho de bovinos mestiços Chianina x Nelore. 1. Fatores envolvidos e estimação de parâmetros genéticos. Ars Veterinária, v.10, p.15-25, 1994.

PEREIRA, E.; ELER, J.P.; FERRAZ, J.B.S. Análise genética de características reprodutivas na raça Nelore. Pesquisa Agropecuária Brasileira, v.37, p.703-708, 2002.

PEREIRA, F.A.; SILVA, M.A. Fatores genéticos e de meio que influem no crescimento de animais mestiços Chianina-Zebu durante o período de aleitamento. Revista da Sociedade Brasileira de Zootecnia, v.11, p.636-649, 1982.

SILVA, M.A.; PEREIRA, F.A. Crescimento e desempenho reprodutivo de animais Zebus e mestiços Chianina-Zebu. Revista da Sociedade Brasileira de Zootecnia, v.15, p.116-123, 1986 a.

SILVA, M.A.; PEREIRA, F.A. Fatores de meio e genéticos que influem no desempenho reprodutivo de fêmeas Zebu e mestiças Chianina-Zebu. Revista da Sociedade Brasileira de Zootecnia, v.15, p.132-141, 1986b.

SILVA, H.M.; SAMPAIO, I.B.M.; VILLALBA, J.J.C. Reprodução em gado de corte. I. Idade à primeira fecundação e período de gestação em Chianino-Nelore. Arquivos da Escola de Veterinária da UFMG, v.31, n.3, p.443-450, 1979a.

SILVA, H.M.; SAMPAIO, I.B.M.; VILLALBA, J.J.C. Reprodução em gado de corte. II. Período de serviço e número de serviços por concepção em Chianino-Nelore. Arquivos da Escola de Veterinária da UFMG, v.31, n.3, p.451-461, 1979 b. 
SIMIONI, V.M. Estudo genético-quantitativo de algumas características reprodutivas na raça Nelore. Jaboticabal: Universidade Estadual Paulista, 2002. 40p. Tese (Doutorado em Zootecnia) - Universidade Estadual Paulista, 2002.

VILlares, J.B. Bovino Chianina no trópico. São Paulo: Associação Brasileira dos Criadores de Chianina, 1975. 205p.

ZAMORANA BUCIO, J.M. Fatores de meio e genéticos na variação de peso e ganhos em peso de produtos resultantes do cruzamento das raças Chianina e Nelore a diferentes idades. Belo Horizonte: Universidade Federal de Minas Gerais, 1979. 66p. Dissertação (Mestrado em Zootecnia) Universidade Federal de Minas Gerais, 1979.
ZILLO, L.R.; OLIVEIRA FILHO, E.B.; DUARTE, F.A.M. Aspectos do desempenho reprodutivo de um rebanho Nelore ligados à precocidade sexual e duração da gestação. In: REUNIÃO ANUAL DA SOCIEDADE BRASILEIRA DE ZOOTECNIA, 23., 1986, Campo Grande. Anais... Campo Grande: Sociedade Brasileira de Zootecnia, 1986. p.360.

Recebido: $16 / 02 / 04$ Aprovado: 23/01/06 